\title{
Sly Syndrome
}

National Cancer Institute

\section{Source}

National Cancer Institute. Sly Syndrome. NCI Thesaurus. Code C84903.

A rare autosomal recessive lysosomal storage disease caused by deficiency of the enzyme beta-glucuronidase. It is characterized by hepatosplenomegaly, skeletal deformities, enlarged head, and mental retardation. 